\title{
Research on Trust-Based Access Control in The Internet of Things
}

\author{
Xiao-hui Cheng ${ }^{1}$, Meng $\mathrm{Hu}^{2}$, Tong Wang ${ }^{3}$, Yun Deng ${ }^{4, *}$ \\ ${ }^{1,2,3,4}$ College of Information Science and Engineering, Guilin University of \\ Technology, Guilin Guangxi, China \\ 1'cxiaohui@glut.edu.cn, ${ }^{2}$ profesor_hu@163.com, ${ }^{3} 1023564574 @ q q . c o m$, \\ ${ }^{4}$ woshidengyun@sina.com
}

\begin{abstract}
The paper established TBRI (Trust-Based RBAC in IoT) model, calculation of trust value as the core in TBRI. It established groups, objects' access threshold, objects' influence and object evaluation, in order to realize accuracy calculation and prevent hackers obtain trust value through malicious repeated operation. Because the subjects in the Internet of things can be used as objects at the same time, TBRI use different trust value calculation formula to enhance the credibility of the trust value.
\end{abstract}

Keywords: Information security, The Internet of things, Access control, Trust

\section{Introduction}

The Internet of things system contains a huge number of users and huge amounts of data, reasonable access control model can effectively improve the security of the data in the system. However, in the original study mandatory access control and discretionary access control should authorize every users' permission by the system administrator, in the face of huge amounts of data is not feasible and dynamic. In order to ensure the security of the data, reduce the complexity of managers assigned permissions, and improve the dynamic access control, access control model based on trust is the key technology to solve the above problems. The introduction of trust value computation in the access control model, the user with permission at the same time has enough trust value to ensure the rationality of its operations, to avoid the legal users illegal operation of data, the illegal users access the data.

\section{IOT Access Control Model based on Trust}

\subsection{Features of IOT Access Control}

IoT compared with existing network communication, in terms of technology and the structure of application layer, the Internet of things has its remarkable characteristics, mainly reflected in the following aspects:

1) A huge number of terminals. IOT realizes something is linked together; each terminal can be as an independent Internet terminal, so according to the development speed of the Internet of things, the number of terminals will be rapid growth.

2) Terminal diversity. Because each terminal can be in the Internet of things so the terminal type is also differ in thousands ways, message communication and transmission between heterogeneous terminals freely is the core issue of the Internet of things.

\footnotetext{
* corresponding author:

Email: woshidengyun@sina.com (Yun Deng)
} 
3) Perceived widely. The Internet of things contains wireless technologies such as RFID technology, discrete terminal will be connected through the perception of Internet of things, It will improve the fineness of the previous connection, enhance network technology to an unprecedented level and expand the scope of perception.

To sum up, in view of the subjects of the Internet of things access control can be accessed as objects. And a variety of terminal in the access control model should be analyzed due to its vast automation terminal in access control model.

\subsection{Formal Description}

The basic elements of access control model are: subjects, objects and access control policy, in today's popular role access control model based on trust, roles, permissions and interaction history are also as fundamental parts. Besides the above elements, there are elements of TBRI as following:

Definition 1 Users' trust value (TRUSTVALUE): $T V=\left\{t v_{i} \mid t v_{i} \in[-1,1], i \in N^{+}\right\}$a property value of the users, trust value $T V$ are calculated by the trust value algorithm based on the session history. When the trust value is higher than the access threshold, permissions are activated.

Definition 2 Interaction time (SESSION_TIME): It record history time of subjects and objects interaction time, calculates current trust value combined with attenuation degree.

Definition 3 Related group (GROUP): $G=\left\{g_{1}, g_{2}, \cdots, g_{n}\right\}$ resources are divided into groups by the similarity of contents and functions, users are divided into groups by the similarity responsibility and identity.

Definition 4 Threshold of objects (THRESHOLD), $T H\left(o_{j}\right)=t h_{j}$ set the threshold according to the classification of objects, when the subject trust value is higher than the object's threshold, the subject can activate the permissions. To avoid the hidden safe trouble made by the role is equal with permissions.

Definition 5 The effect degree of objects(INFLUENCE), $I N\left(o_{j}\right)=i n_{j}$ in order to avoid the subjects accumulated trust value by repetitive operation, so the effect degree of objects are assigned depending on the trust level of objects, irregularities operations reduce trust value, legal operations improve trust value. The effect of trust and the access threshold has a certain impact $f\left(i n_{j}\right)=t h_{j}$.

Definition 6 Evaluation coefficient of objects $. \lambda\left(s_{k}, o_{m}\right)=\lambda_{k m} \cdot \lambda_{k m}$ is the evaluation coefficient of the object $o_{m}$ for the subject $s_{k}$, when fully trust $\lambda_{k m}$ is 1 , distrust $s_{k}$ is 0 , completely refused access is $-1, \lambda_{k m} \in[-1,1]$. When the operation evaluation coefficient is $\lambda_{k m}$, combined with the effect degree of objects $i n_{m}$ of object $o_{m}$, the impact of trust value is $\lambda_{k m} * i n_{m}$. Through operating the objects, the trust value in the range of $\left[-i n_{m}, i n_{m}\right]$.

\subsection{Calculation of Trust Value}

Calculation of trust value is mainly composed of three parts, respectively is the direct trust and recommendation trust and comprehensive trust value.

2.3.1 Direct Trust: Direct trust (Direct trust, DT) calculate trust value based on the interaction history between the subjects and objects, because only in view of the object which had interaction history with the subject. The characteristics of the direct trust: update trust value with decay time,$t_{o}$ is the interaction time, $t_{n}$ is the time of apply for 
access, $\sigma$ is the attenuation factor, attenuation degree $\rho\left(t_{n}, t_{o}\right)=e^{-\sigma\left(t_{n}-t_{o}\right)}, T V(k, m)$ is the trust value of subject $s_{k}$ to access object $o_{m}$ trust value, the formula of direct trust is :

$$
D T\left(k, m, t_{n}, t_{o}\right)=e^{-\sigma\left(t_{n}-t_{o}\right)} * T V(k, m)
$$

)

Direct trust represents ultimately trust value of last time, it is the most authoritative reference value, but due to the direct trust contains only one object, and there is no guarantee that after the last interaction the subject still a legitimate users, Time attenuation represents the trend of trust value, its general trend is shown in figure 1.

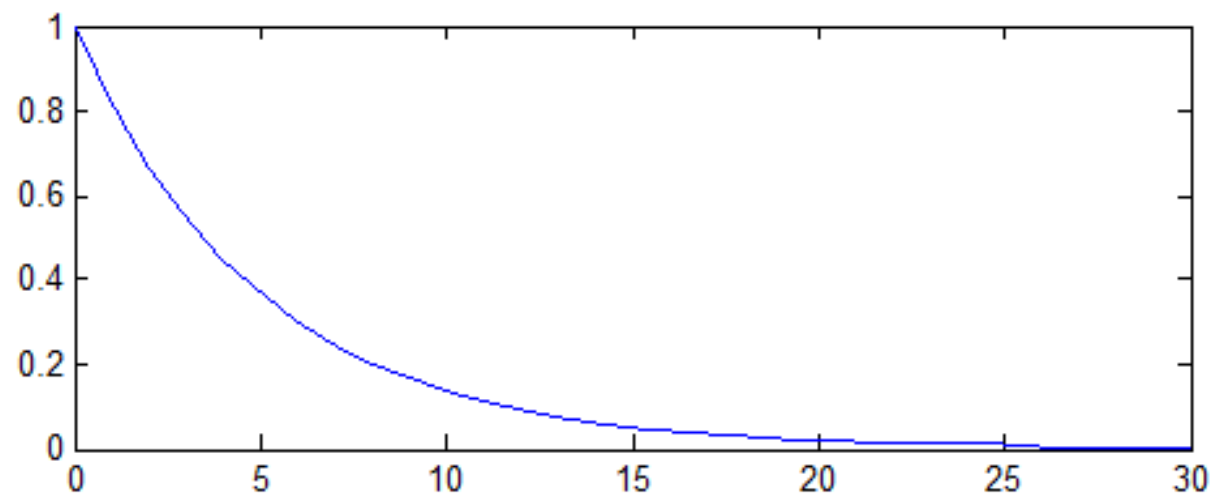

Figure 1. The Direct Trust Trend

2.3.2 Recommendation trust: Recommendation trust (Recommendation trust, RT) is when the subject access objects the other objects according to the history of interaction recommend the subject. When the subject has irregularities history with other objects, the recommendation trust value is negative. When the history is legal operations, the recommendation trust value is positive. In the Internet of things, the subject can access to other objects, so the calculation of recommendation trust value should be distinguished according to the categories of subjects or objects.

When the subject is of a single attribute, the subject is an independent program or a user. The subject is $s_{k}$, the object is $o_{m}, o_{j}$ is the object which recommend $s_{k}$ to $o_{j}$, $n$ is the total number of objects, $R T(k, m)$ is recommendation trust value. When the subject is a user or an independent program, recommend trust computing is mainly based on the direct trust between the object and the subject, and according to the correlation of them to calculate weights of all the direct trust value.

When the subject is a natural person or an independent program, the recommendation trust formula is:

$$
R T(k, m)=\sum_{j=1}^{n} \frac{r v_{m j}}{\sum_{j=1}^{n} r v_{m j}} D T\left(k, m, t_{n}, t_{o}\right)
$$

The double attribute subject, the subject has object properties. $m$ is the object, $j$ is the recommended object number, $p$ is the total number of objects and the objects come from the same group, $g$ is the number of objects come from related groups, $A L(k, m)$ is the trust value algorithm in this context, $\lambda_{k m}$ is the evaluation coefficient of objects. $\alpha$ is the recommend weight from the same group,$\beta$ is the recommend weight from the other groups, and meet $\alpha+\beta=1$ and often $\alpha>\beta$.

When the subject has double attributes, recommendation trust values is combined by 
the same groups' objects and the rest of the group's objects, and limit effect scope of each object, thus avoiding the subject malicious accumulate trust value. The default correlation $r_{i j}$ is 1 . When the main body for the resource, recommendation trust value computation formula is:

$$
R T(k, m)=\alpha * \sum_{j=1}^{p} \frac{\lambda_{k j} i n_{j}}{p} A L(k, j)+\beta * \sum_{g=1}^{q} \frac{\lambda_{k g} i n_{g}}{q-\sum_{g=1}^{n} \frac{0}{r v_{m g}}} A L(k, g)
$$

Rule : When $r v_{i j}=0, \frac{0}{r v_{i j}}=1$ 。

Recommendation trust value formula only represents the calculation steps and the influencing factors, the specific recommendation trust value algorithm is selected according to the specific context, in this paper, $A L(k, m)$ selects EigenTrust calculation algorithm.

So the recommendation trust value consists of recommendation trust value formula and recommendation trust value algorithm, the algorithm is applied in the formula to calculate recommendation trust value.

2.3.3 Comprehensive Trust Value: Comprehensive trust is the ultimate trust value of the subject, comprehensive trust is composed of direct trust and recommendation trust, $\omega_{1}$ and $\omega_{2}$ are the weights of direct trust and recommendation trust, and $\omega_{1}+\omega_{2}=1$. The comprehensive trust formula is:

$$
T V(k, m)=\omega_{1} * D T\left(k, m, t_{n}, t_{o}\right)+\omega_{2} * R T(k, m)
$$

The weight of direct trust and recommendation trust are determined based on specified context, in this paper, in the common context and unconditional restrictions, rules $\omega_{1}=\omega_{2}=0.5$.

\section{TBRI Safety Experiment}

In the past access control model, the sever adopted the accumulation of trust value to improve the trust value of the subject, so will produce the problem that subject can improve its trust value when malicious repeated operation, cause the classification of the higher object cannot get effective protection. Therefore how to reasonable control calculation trust value of repeat access an object, it plays an important role in IOT security.

In safety experiment, through repeated operation of objects, to judge whether TBRI can effective accumulate trust value and avoid malicious subjects repeated get higher trust value through repeated operate same objects.

TBRI set up three different subjects and 10 objects which have different characters as a test case and matching them one by one. Each time the subject operate the same operation, a total of 10 groups operation and 300 times of apply for access. TBRI recorded the change of the three subjects' trust value, monitoring the subjects whether can get higher trust value through repeated operation and keep trust value rising trend.

According to the above plan to perform the experiment, in each group of experiments, the average trust value of 10 objects as the object trust value of the group, the running results as shown in figure 2 .

In Figure 2, the 10 subjects' trust value is improved in the first few group operation; the growth rate slowing down in the middle of process, in the last groups the trust value remains unchanged.

The conclusion can be drawn: because the effect degree of object is set, any object increase the degree of an subject's trust value with a certain limit, and will not increase 
trust value because of repeated operations. It can effectively obtain the trust value through malicious repeated operations.

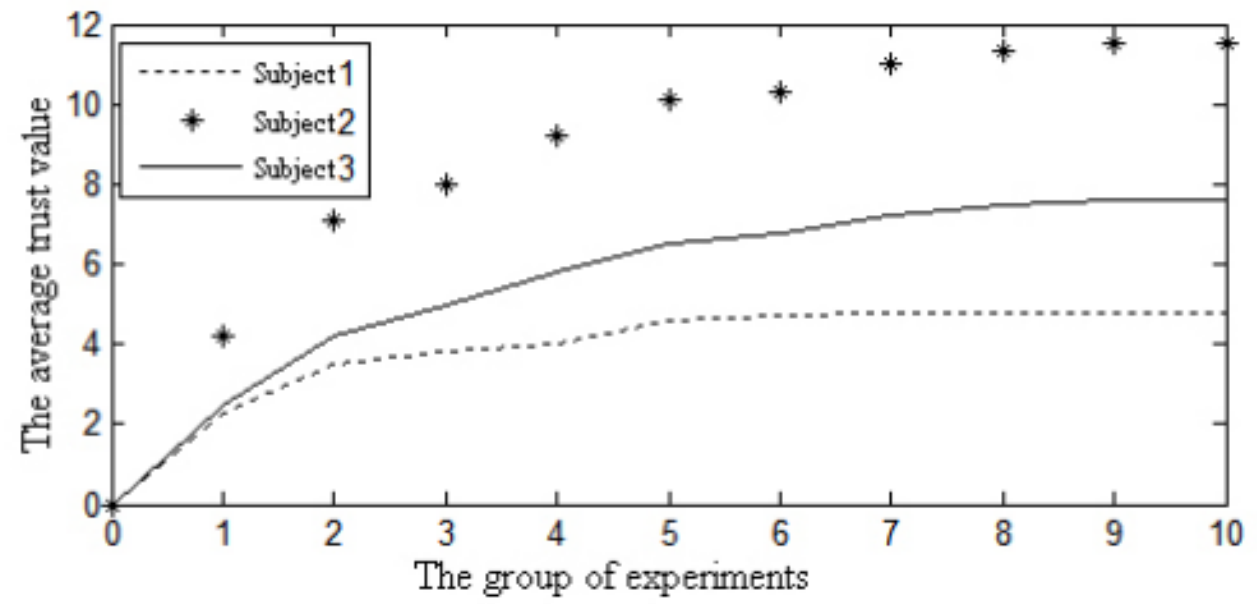

Figure 2. Trust Value Trend

\section{Conclusion}

TBRI can calculate trust value based on whether the subject can be access as an object, increases the credibility of trust value. At the same time, due to the coefficient degree and the influence of objects are set, they can effectively calculate the trust value, and the subject can be prevented accumulate trust value through malicious repeated operations. For the future of the Internet of things access control research has certain reference function.

\section{Acknowledgements}

As the research of the thesis is sponsored by National Natural Science Foundation of China (No: 61262075) and major scientific research project of Guangxi higher education (No: 201201ZD012), we would like to extend our sincere gratitude to them.

\section{References}

[1] Sun E, Zhang X, Li Z. The internet of things (IOT) and cloud computing (CC) based tailings dam monitoring and pre-alarm system in mines[J]. Safety science, 2012, 50(4): 811-815.

[2] GubbiJ,BuyyaR,MarusicS,et al.Internet of Things (IoT): A vision, architectural elements, and future directions[J].Future Generation Computer Systems,2013,29(7):1645-1660.

[3] Atzori L,Iera A,Morabito G.The internet of things: A survey[J].Computer networks,2010,54(15):2787-2805.

[4] Gusmeroli S, Piccione S, Rotondi D. A capability-based security approach to manage access control in the Internet of Things[J]. Mathematical and Computer Modelling, 2013, 58(5): 1189-1205.

[5] Sarma A C, Girão J.Identities in the future internet of things[J].Wireless personal communications, 2009,49(3):353-363.

[6] Tan L, Wang N. Future internet: The internet of things[C]//Advanced Computer Theory and Engineering (ICACTE), 2010 3rd International Conference on. IEEE,2010,5:V5-376-V5-380.

[7] Laranjo I, Macedo J, Santos A. Internet of things for medication control: Service implementation and testing[J]. Procedia Technology, 2012, 5:777-786.

[8] Gouglidis A, Mavridis I. domRBAC: An access control model for modern collaborative systems[J]. Computers \& Security, 2012, 31(4): 540-556. 


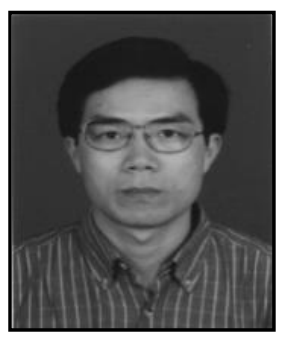

\section{Authors}

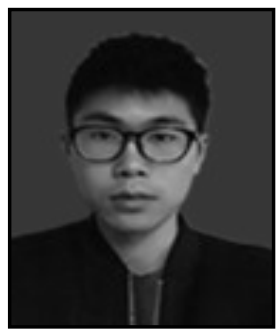

Xiaohui Cheng, Professor, College of Information Science and Engineering, Guilin University of Technology. Current researches are on information processing and embedded system.

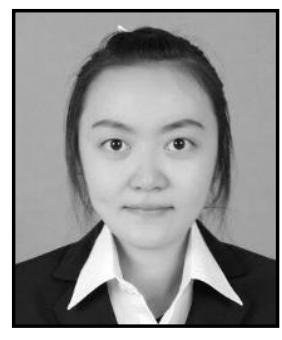

Meng Hu, Graduate Student, College of Information Science and Engineering, Guilin University of Technogy. Current researche is Trust-Based Access Control in The Internet of Things, The Internet of Things Technology

Tong Wang, Graduate Student, College of Information Science and Engineering, Guilin University of Technogy. Current researche is Trust-Based Access Control in The Internet of Things, The Internet of Things Technology

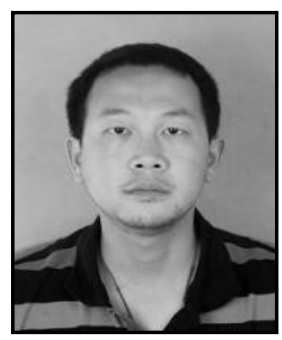

Deng Yun, College of Information Science and Engineering, Guilin University of Technology, Guilin, China. Current researches are on Internet of Things and embedded system. 